\title{
"I Can’t Lie Anymore!": The Implications of Location Automation for Mobile Social Applications
}

\author{
Sami Vihavainen, Antti Oulasvirta, Risto Sarvas, Helsinki Institute for Information Technology HIIT / \\ Helsinki University of Technology TKK
}

\begin{abstract}
Human factors research has shown that automation is a mixed blessing. It changes the role of the human in the loop with effects on understanding, errors, control, skill, vigilance, and ultimately trust and usefulness. We raise the issue that many current mobile applications involve mechanisms that surreptitiously collect and propagate location information among users and we provide results from the first systematic real world study of the matter.

Our observations come from a case study of Jaiku, a mobile microblogging service that automates disclosure and diffusion of location information. Three user groups in Finland and California used Jaiku for several months. The results reveal issues related to control, understanding, emergent practices, and privacy. The results convey that unsuitable automated features can preclude use in a group. While one group found automated features useful, and another was indifferent toward it, the third group stopped using the application almost entirely. To conclude, we discuss the need for user-centered development of automated features in location-based services.
\end{abstract}

Index Terms - Automation, human factors, location information, mobile social applications, privacy, user-centered design

\section{INTRODUCTION}

A UTOMATION is utilized extensively in human activities ranging from product manufacturing to chemical and power plants, space vehicles and robots, heating and air conditioning, business systems, medical devices, home appliances, and stand-alone computers. Human factors research has shown that automation is a mixed blessing. It changes the role of the human in the loop with effects on understanding, control, skill, vigilance, and ultimately trust

This study was part of the Finnish Funding Agency for Technology and Innovation (TEKES) project Täky. Antti Oulasvirta was supported by the Academy of Finland project Amoveo.

S. Vihavainen is with Helsinki Institute for Information Technology HIIT / Helsinki University of Technology TKK P.O. Box 9800, FIN-02015 HUT, FINLAND (phone: +358 44348 2517, e-mail: sami.vihavainen (at) hiit.fi).

A. Oulasvirta is with Helsinki Institute for Information Technology HIIT / Helsinki University of Technology TKK, P.O. Box 9800, FIN-02015 HUT, FINLAND (e-mail: antti.oulasvirta (at) hiit.fi).

R. Sarvas is with Helsinki Institute for Information Technology HIIT / Helsinki University of Technology TKK, P.O. Box 9800, FIN-02015 HUT, FINLAND (e-mail: risto.sarvas (at) hiit.fi). and usefulness [21]. Many present-day mobile applications involve mechanisms that surreptitiously collect and propagate location information among users. The raison d'etre for location automation in these applications is productivity: a mobile user does not have the time and resources to manually post and update her location information. Moreover, automation is always a temptation. If the system can capture and share more information, why not do it?

But what are the implications of introducing location automation into mobile applications that are inherently social by character? In this case, the "process" that is being "controlled" here is social by nature, not safety- or performance-related. The implications of automation to computer-mediated social interaction have not been systematically addressed although the question is of utmost importance for information and communication technology. It can be justifiably predicted that the implications will go beyond productivity-related issues. This area of interest, especially from non workplace communication perspective, has recently also brought up for example by [19] as part of important future $\mathrm{HCI}$ research.

We consider this issue relevant to the Mobiquitous community, because whenever we design middleware or UIs for automatic location-disclosure, we subscribe to a model of automation that may or may not be suitable for users. The user does not "see" beyond the immediate information in the user interface, yet important decisions on self-disclosure are determined by the automation. There are many open questions, like: Which models of automation are acceptable in location disclosure in non workplace related communication? How do they affect interaction and use? How can control mechanisms be designed for the user?

We present a case study of Jaiku to provide first data on this matter. Instead of general aspects of user experience or usability of the user interface, we focus on automated features that in a way "exist" beyond the user interface in the system that collects and propagates location information to other users. Our goal is to shed light on three broad questions, the first two we believe are unique to mobile social applications. The third has been studied extensively in human factors, but not in this application context: 
1. Use of automated features in mobile social applications

2. User response to automation

3. Users' understanding of the logic of automation

As a case, we study location automation in an application called Jaiku. Fig. 1 represents Jaiku's user interface. In a nutshell, Jaiku is a mobile awareness service that allows a group to share textual status updates that are associated with automatic location information. Moreover, a number of awareness cues are provided. At first blush, these two automatic features look quite harmless. Under the surface, however, both applications involve quite complicated automation to control the construction, propagation, utilization, and visualization of these data. We start the paper by analyzing these models, leveraging the levels of automation framework from human factors.

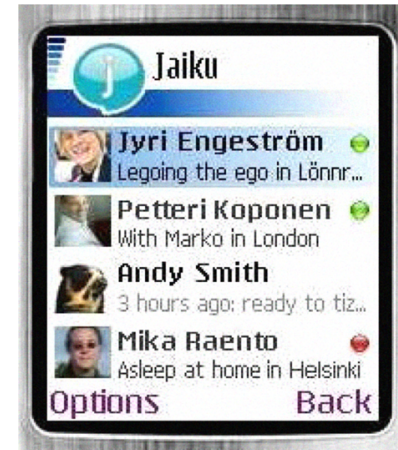

Fig. 1. The user's perspective to Jaiku. The UI shows contacts' status messages with location cues.

The next main part of the paper presents the study and its results. By studying three user groups' use of Jaiku our goal is to gain a more general perspective to the phenomena, instead of pooling results from an individual user group. Interviews and $\log$ analysis were used as the main methods of data collection. At the end of the paper, we return to the challenges that automation pose to mobile applications.

\section{A. Overview of Jaiku}

The next sections outline a detailed automation model Jaiku uses, but at this point we want to provide a general overview of the system to concretize and contextualize our work. Jaiku (see Jaiku.com) is a Nokia S60-based mobile awareness service built on the ContextPhone platform [17]. There are three concepts of relevance here, which also dominate the UI of the Jaiku mobile client (see Fig. 1):

1. Status messages: Users can post status messages of 140 characters that are viewable by their contacts using a mobile client or Internet browser (microblogging). In our studies we focused on within-group use, although Jaiku allows also for public publishing. This is not an automated feature, as its contents are fully usercontrolled. The automated features are meant to support status messages and in a way contextualize them.

2. Location label: Parallel to the status line there is location information which shows a label for the user's GSM cell ID. This feature is automated (partially), as we will discuss in a forthcoming section.

3. Awareness cues: In addition, Jaiku provides a separate screen with real-time sensor-derived indicators (awareness cues) such as online status, alarm profiles, number of other people in proximity and the next calendar event. This feature is also automated (partially), as we will discuss in the forthcoming section.

\section{RELATED WORK}

\section{A. User studies}

A growing body of good user studies of location awareness application is emerging [5], [7], [8], [9], [10], [15], [22]. Many of these are made in workplace context and not in socially more complex leisure environments. Many of these papers also note briefly that automatic features were used or noted by the users, but only a handful give the issue more weight. In what follows, we go through them in more detail.

Barkhuus et al. [5] studied an awareness system called Connecto that allows users to tag locations and share them, automatically or manually, on a mobile phone. The goal of the study was to understand how location awareness would work within a close-knit group of friends. They recruited two separate groups of friends. However, they did not do any comparison between the results from each group. Their users manually controlled, otherwise automatic, location mostly when they needed to "freeze" the location for others. The main reaction reported related to better communicativeness that can be achieved via manual overriding. The users were not reported having shut off the automatic disclosure for reasons of privacy.

Brown et al. [7] studied a system called Whereabouts Clock. Whereabouts Clock is a desktop terminal, a 'clock', which shows the location of each family member based on their mobile devices' current locations. The authors' starting point is that a key aspect of family activities is to know other members' whereabouts and routines. The system was used by five families, all of whom used the clock quite actively and reported no significant problems in its use. However they did not present any specific results between the families or explored the social boundaries of the system with other types of groups. Moreover, location-disclosure was of low fidelitythe clock only enabled one to see if another member is physically at home or not.

Consolvo et al. [8] conducted an interesting study where they studied whether and what the users are willing to disclose about their location to social relations. They conducted a three-phased formative study. Their results show that most important factors are: who is requesting, why the requester wants the location information, and what detail would be the most useful to the requester. However, they did not do any real world user trial on automation related questions.

Harper [9] used Active Badge location technology for studying social organizations of two research laboratories. He states that the one's role within the moral order of the 
organization affects on the acceptability of a new technology in workplace organization. For example in workplace the information on the location may be a status quo in case of the receptionist, but not in case of an individual researcher. However, in his study Harper does not concentrate on automation and also focuses on workplaces with formally specified hierarchy and which social relations are more static than leisure time social groups'.

Iachello et al. [10] studied the awareness application Reno, which allows querying the locations of friends and disclosing one's own location to them. In addition to non-automated (i.e., fully manual) disclosure, location names can be set to be revealed to selected contacts automatically, or upon entering a pre-specified location. The results show that participants did not use automatic features almost at all. The main reason stated is that they did not fully trust they would work properly and they did not feel a subjective need for setting up the automatic features. However, only one out of eleven expressed privacy-related concerns as the reason. The authors' conclusion was that there is no need for automatic location disclosure in Reno.

Want et al. [22] studied a building based location system Active Badge in office working environment. They used wearable electronic ID badges to automatically disseminate location of the participants. The building had detectors that recorded participants' location in every 15 seconds. Using a computer user was able to locate the participants based on map or a textual interface. The system also showed who were in the same room with each other and the nearest telephone number. They report that the system had many advantages such as the incidence of telephone calls not reaching the correct person dropped. In addition they also reported problems related to privacy. They reported that to most people first reaction for personal location system was horror but that after 2 weeks mandatory use many continued using the system. However, Want et al. studied the system in workplace environment which lacks the social complexity of everyday life and does not take into account such diverse areas as play and expressiveness.

Taken together, automated features have been associated with mainly to four issues: understanding, user needs, communication, and privacy. However, previous studies do not recognize that all the four issues are explicitly related to the level of automation in the system's features. Thus there was a need for systematically study automation in social mobile media. The results do not lend strong evidence for general usefulness of automated features in either, for example usefulness with microblogging - a finding that our study can elaborate. By comparing three user groups, only one of which preferred the automation of Jaiku, we wish to illuminate how group structure and activities affect which forms of automation are useful and acceptable.

\section{B. Location automation}

In this subsection, we do not intend to provide a thorough review of the state of the art. Instead, by providing a few examples, we want to illuminate the recent surge in the number and variety of location automation solutions.

Much of the technology research in these types of services focuses on better ways of producing accurate location information using existing network infrastructures (e.g., GSM, GPS and Wifi). See, for example, [11] and [23] for location determination in WiFi networks. The production of accurate location has also strong business incentives, and different technologies and algorithms for generating accurate, fast and reliable location information are turned into business (see, e.g., Skyhook wireless). Junglas and Watson [12] list three challenges for the adoption of location-based services: more accurate location information, faster response times, and privacy concerns raised by users. As we will attempt to argue later, the model of automation will affect how much users put weight to all of these concerns.

Against this backdrop, our contribution to research in location-based services is in studying location information in a mobile social application. In other words, the use of location information in Jaiku is primarily social interaction between people, not for example, a search function (e.g., show me the closest gas station).

TABLE 1. LEVELS OF AUTOMATION ACCORDING TO SHERIDAN [20], [21]

1. The computer offers no assistance; the human must do it all

2. The computer suggests alternative ways to do the task

3. The computer selects one way to do the task and asks for human approval

4. The computer allows the human a restricted time to veto before automatic execution

5. The computer executes the suggestion automatically, then informs the human

6. The computer executes the suggestion automatically, informs the human if asked

7. The computer selects the method, executes the task, and ignores the human.

\section{UNDERSTANDING AUTOMATION IN MOBILE SERVICES}

Since we talk about automation of location information, it is necessary to start by explaining our terminology. By location information in Jaiku we mean the information displayed in the user interface right after the status updates (see Fig. 1). The location information is identified by the word "in". For example, in Fig. 1, for the user "Petteri Koponen" the location information is "London". We do not attempt a broader definition at this point. Conceptually the location information in Jaiku is metadata about the user and the status line. It is technically coupled with the user's phone: it changes as the user's phone's cellID changes, or if the user changes the label manually. Visually the location information is embedded into the status line as it is appended to the end of it. 


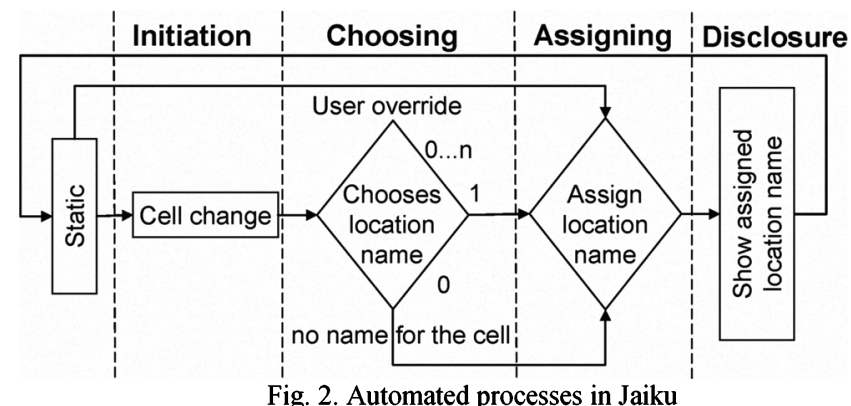

A. Automation models in Jaiku

Sheridan [20] has proposed a level of automation framework. The levels range from no assistance from a computer to a computer is in full control without informing the user (Table 1, previous page). We believe this framework suits well the description of how automation works in Jaiku. Four consecutive processes can be identified:

1. Initiating location information change,

2. Choosing location label,

3. Assigning location label, and

4. Disclosing location label.

All of these can be automated at any of the seven levels. Fig. 2 presents the flow chart of the automated processes. To contextualize the results of the user study, it is necessary to describe in some more depth the complex workings of location automation in Jaiku.

\section{1) Initiation of location information change}

The first part of location automation in Jaiku is the initiation of the location information change. In Jaiku, this part of the process is triggered whenever the phone's cell tower changes. The user, of course, cannot know when this happens as these cells are not visible in the environment. However, with practice the users may learn where cell boundaries are. Because the user has practically no means to know when a cell tower changes, she/he has practically no control over the initiation. Initiation triggers the next steps: choosing, assigning, and distributing.

\section{2) Choosing location label}

As mentioned above, the location information is acquired using the cellIDs. For each cellID and username pair, there are $0 \ldots$ options for location labels. If the user has previously input a label for the cellID at hand, then that single label is the result of the analysis. If the user has input no label for the given cellID, then the user's contacts network is searched for whether any user within the network has given a label. If no one in the user's network has given that cellID a label then the result is null. If there is several labels then the system chooses one (the algorithm is unknown to us). Importantly, the user has no means to affect this process other than shut down Jaiku. It is unlikely that the users understand the logic of choosing, as we will discuss later.

\section{3) Assigning location label}

The third part of the process is the assignment of metadata to a username. In Jaiku, this is fully automatic unless the user overrides this by typing in a location label. Thus, manual overriding is possible. If the user does not choose to override it, the metadata chosen in the previous process is automatically assigned to the username. If the user chooses to type in a label, the typed label is assigned and stored into the system's database. Importantly, the user has to actively initiate this because Jaiku in no ways prompts the user to write anything. Next time the user's Jaiku enters the cellID it will automatically choose the typed label.

\section{4) Disclosing location label}

The last part in this process is the disclosure of the assigned label. It means that the application sends the assigned label to the user's contacts' Jaiku clients; not directly, but through the Jaiku server. Each of these clients then updates the UI (Fig. 1) to show the label in association with the status line. This is fully automated, the user has no means to control when to disclose and to whom. Shutting down the application and manually removing entries from the contact book are the only options for control here.

\section{B. Speculating broader social implications of automation}

The Before moving on to our study, we want to provide some background on possible social consequences of location automation in light of studies done in human-computer interaction. These are speculations but relevant because they guided the calibration of our data collection methods.

In Jaiku, the automatic disclosure of personal information can be speculated to have effects on three mobile device mediated activities. The findings of the study show effects (negative and positive) relating to the two first ones. First, mobile phones cater a handful of channels through which users coordinate shared activities and [4], [11], as part of that, disclose information about themselves. In this activity, automatic disclosure may have the advantage of saving a person from continuously updating others with current location and plans via SMS or calls [15]. However, if the disclosed information is not useful, it may be add to information overload on the side of the receiver. Second, mobile phones are one channel in which people engage in negotiation of privacy. Palen et al. [16]] write: "The boundary between self and other is destabilized when phone users assume that they are without an audience." Continuous disclosure about oneself may erode users' ability to control this process and result in that they reject the system, inhibit its use, turn off automatic disclosure or establish extraneous practices to repair the damage done. Third, mobile device communications contributes to maintenance and deepening of social relationships. A significant factor in this process is the reciprocation of self-disclosure. Communicators tend to model the level of intimacy in each others' disclosure, and people who do not respond with reciprocal disclosure, or who disclose too much, are generally disliked [2]. Automatic 
disclosure may be too rigid, reveal too much information or too little, and thus may have to be "repaired" explicitly by the conversant. By the same token, continuous automatic disclosure of one's "true" activities may conflict with the images one desires to convey.

\section{THE STUdY: ThreE FIELD TRIALS OF JAIKU}

Three groups (students, birders, hipsters) were recruited in Finland and California, and they used Jaiku for two months. A mix of quantitative (content logs) and qualitative (interviews) methods were used in order to gauge both interaction on the phone and subjective views on automation.

\section{A. User groups}

1. The Students were a group of 5 men and 5 women, ages from 18 to 20 years, living in the Helsinki metropolitan area (Finland). They spent time together in school and in their freetime. During the trial, they had several joint events, such as a ferry trip to Stockholm. Many of them participated in a preparation course for university entrance exams. The participants were not particularly tech savvy but were moderately fluent users of cell phones and the Internet.

2. The Birders were a group of 7 men and 1 woman, ages from 18 to 64 . Birders were an interest group, all belonging to a bird watching club in Helsinki. Four Birders knew each other beforehand, but the rest had not met prior to the study. During the study, many Birders traveled frequently in southern Finland. Almost all communications within the group was about bird watching. All were active users of a national bird observation service (Lintutiedotus) that distributes information about recent observations via SMS.

3. The Hipsters were a group of 4 men and 4 women, in their 30's and living in the Bay Area, California. Three lived together and there were 2 dating couples. All shared a similar life style that is commonly called "hipster" and spent plenty of time with together. The Hipsters were not particularly tech savvy, except two men who used the Web in their work. All members were fluent in using cell phones and services on the Web, such as Web shopping.

\section{B. Procedure}

All participants were provided with Nokia N70 phones and free data plans. They were introduced to the application in pretrial group sessions. While our instructions focused on the use of the application and its UI, we also had to explain basics of the automated features, for example, automatic location diffusion. We encouraged them to use or to at least try the features (e.g., manual overriding of location labels).

Two weeks after the start each user was contacted to check that everything was working. All groups used the application for two months. No other reward than free data plans for the time of the trials was provided.

\section{Data Collection}

1. Interviews. The users were interviewed individually about their social networks, communication practices, how they used Jaiku, what kind of feelings they had about the application and how they used and understood the automated features. We asked them to express general opinions but also tell concrete, real episodes of use. The interviews took place at users' homes, work places, or schools. 41 hours of interview data were gathered. The protocol had the structure described in Table 2 below.

TABLE 2. THE INTERVIEW PROTOCOL.

\section{Warm up discussion}

2. General communication with other people (e.g. Who do you communicate with on daily basis?)

3. Jaiku in general(e.g. Jaiku in your own words?)

4. Usability (e.g. was it hard to learn to name locations?)

5. Privacy (e.g. How do you like the fact that your contacts can see your location?)

6. Presence line (e.g. Why have you written presence lines?)

7. Location (e.g. Tell me about the last time you wrote your location in Jaiku)

8. Checking contacts' information (e.g. Tell about the last time you checked your contact's location)

9. Effect of Jaiku on use of other comm. channels (e.g. What was Jaiku's effect on SMS use?)

10. Needs (e.g. Has Jaiku been useful to you?)

11. Final (e.g. How would you develop Jaiku?)

2. Logging. Logging consisted the data described in Table 3. A shortcoming in our logging is that we could only access location labels as they were sent with the messages, but not when users manually updated their location labels. This shortcoming precludes a thorough analysis of users' practices in manually updating/overriding labels. However, most of the time when a user updates location manually, it is done with the purpose of disclosing it with the message. Therefore, most updates are caught in our data.

TABLE 3. DATA LOGGED IN THE USER TRIALS.

1. User ID

2. Written presence line

3. Location where the presence line was written

4. Time and date the presence line was written

\section{V.FINDINGS PART 1: USE OF AUTOMATED FEATURES IN CONCERT WITH MESSAGING FUNCTIONALITY}

In this section, we describe the usage of the application's communication functionality, its main designed purpose, from the perspective of the automated features. Statistics on use and opinions convey differences among the groups.

\section{A. Sending messages}

Jaiku was a new application to all users, and there was a learning phase in all groups to establish a way to use the system. The basic concept of an awareness system (an 
application that enables the user to communicate her location and activities to her friends) was clear for all users.

TABLE 4. SYSTEM ACTIVITY IN THE THREE GROUPS.

\begin{tabular}{|c|c|c|c|c|}
\hline & Students & Birders & Hipsters & SUM \\
\hline Users & 9 & 8 & 8 & 25 \\
\hline Period & $4-6 / 07$ & $4-6 / 07$ & $9-12 / 07$ & \\
\hline $\begin{array}{l}\text { Sent Jaiku } \\
\text { Messages }\end{array}$ & 1004 & 550 & 53 & 1607 \\
\hline $\begin{array}{l}\text { Locations } \\
\text { written }\end{array}$ & 112 & 122 & 11 & 245 \\
\hline
\end{tabular}

During the two months, the 25 users sent 1607 messages and wrote 245 location tags. Table 4 shows the Jaiku activity of the groups.

The Students were the most active group. During the first month they used Jaiku mostly for messages to the whole group, typically containing insider comments, and telling about whereabouts and doings. During the second month, Jaiku was used also for one-to-one messages, despite the fact that they were visible to the whole group. They wrote locations frequently in Jaiku, and said they enjoyed following their others' locations.

The Birders were relatively active Jaiku users sending 550 messages and writing 122 locations. In the beginning they used it mainly for reporting birds. Many of the locations named were bird watching places or names of towns in the visited areas. Later on they started to write also about topics not related to their hobby because Jaiku was not perceived as efficient for bird reporting as the existing SMS service.

The Hipsters were the most passive user group, sending only 53 messages and writing 11 locations. Especially in the beginning, many of them had concerns about privacy, saying that it felt "creepy" that Jaiku automatically tracks them. In addition, a few felt that it was unnecessary to be connected more than they already were through other means. Also, many did not get used to the battery drainage. For these reasons, which we will elaborate later on, some of the Hipsters switched it off.

\section{B. Intentions to control location labels}

We interviewed the users on how they control location diffusion and determine the contents of location labels. Generally, the users spent little effort to control the automation.

The Students told us that the location information of Jaiku was useful and they were interested in each others' locations. They named 112 locations. The main uses they mentioned were 1) coordination and 2) having new opportunities for ad hoc encounters, as in the following passage:

"If I see that [participant] is 'in [library A]' and I'm in [library B], I just write 'lets get some food' [to Jaiku's presence line]. If he doesn't notice I can text or call him" - M18

They hardly ever overrode the automatic locations, except for a few occasions, often for the reason of increasing informativeness:

"I changed [name of a building] to [a restaurant in the same building]. I think that tells more for this group." - F19

For Birders and Hipsters, the disclosure of movements in real-time was not interesting. The Birders did not have any shared activities outside bird watching, and even that was not an activity done together. Nevertheless, they did name 122 locations, but this was because they traveled quite actively in Southern Finland. Moreover, the naming of locations dropped significantly after the first month. Even though the Hipsters were good friends and spent time together, they found hardly any use for the location information in Jaiku. The group named only 11 locations.

It seems like the automated location disclosure, together with the presence line, was useful only for the Students who knew each other quite well and interacted with each other frequently. Contrary to other groups, many of their daily activities were shared and automatic disclosure contributed to the coordination of mobility and communication relevant in these activities. For the more heterogeneous groups, Birders and Hipsters, automatic location had little value or use and therefore, they spent little time controlling it.

\section{Enhancing communicativeness with manual overriding}

A central feature of Jaiku is that users can always type their own location label which will override the automatic ones. To study how this happened, we categorized the location labels according to the (subjective) size of the geographical area they referred to. This informed us if the cellID location technology is accurate enough for these uses from the perspective that labels have communicative functions.

TABLE 5. GRANULARITIES OF LOCATION REFERENCES

\begin{tabular}{|lllll|}
\hline & $\begin{array}{l}\text { Stu- } \\
\text { dents }\end{array}$ & $\begin{array}{l}\text { Hip- } \\
\text { sters }\end{array}$ & $\begin{array}{l}\text { Bird- } \\
\text { ers }\end{array}$ & Total \\
Area $<$ neighborhood & $55 \%$ & $36 \%$ & $27 \%$ & $\mathbf{4 0 \%}$ \\
\hline
\end{tabular}

\section{1) Categorization of location label granularity}

We created and applied a simple categorization for the label data. All user-created labels were categorized according to granularity, i.e. the size of the geographical area the label refers with the simple split point:

1. $\quad$ area $\geq$ neighborhood

2. area $<$ neighborhood.

For example, location names like "home" and "library" were categorized in the second category. However, some labels, like the name of a tourist resort did not fit well in this "urban" categorization. The amount of non-categorizable items was small, though $(\sim 2 \%)$.

\section{2) Pin-pointing with more accurate labels}

Table 5 reports the results of this analysis for Jaiku. This analysis shows that the locations written by the users were often quite specific. Jaiku's UI gives users two categories of 
places to name: "neighborhood" and "city/region". However, analysis of the user created names showed that $40 \%$ of the written locations referred to a location more specific than neighborhood. Perhaps unsatisfied with the communicative effect of their location labels, the participants overrode the GSM cellID based automatic with quite specific locations (e.g., "home", "cafe", "library"). Unsurprisingly, places that were visited often or considered important had a more specific label:

'I have named places I visit more often. There is 'home', there is 'gym' and there is 'Prisma' [supermarket]" - F19

The Students named locations more precisely than the two other groups. The Students had specific places known to everyone in the group, which was probably a factor in their detailed labeling. In contrast, the Birders were mobile and traveled around the southern part of Finland to various bird watching places and their group had very little shared history. Therefore, their labeling was not that detailed.

\section{3) Non-location referencing}

We then examined in a second exercise to which extent written labels refer to something more meaningful than geographical areas, for example, 'home' or the name of a cafeteria. Altogether 36\% of named locations had another meaning than a geographical area.

There were a few cases where a location label made visible the event the user was participating in. For example, during the trial most of the Students were taking a course together and the location of the place was named by one of them as "[teacher of the course]". However, for an outsider, the location "[teacher of the course]" would have been ambiguous. Another example is the "[name of an office building]" that was changed to "[name of a pub in the building]". The name of the pub told more about the context of the person than the name of the building.

\section{D.A note on the probable effect of audience}

From the two above analyses, referring to "insider information" in labels worked in the small groups. Many participants told that if the location labels in Jaiku were disclosed outside the group they would use more general names:

"If there would be others (outside the group) I would not name the place as [the university the user was going to applying], I would just write the real name of that place. It is after all [ the institute where the participant was taking a preparation course before applying to the university]" - M19

Thus, our observation that the automatic location labels were often "enhanced" with manual ones is most likely partially explained by the nature of the user groups.

\section{FINDINGS PART 2: USER RESPONSE ON AUTOMATION}

In this section we characterize the user groups' experiences on the usefulness of automation and its perceived on their shared activities. Two out of three user groups thought that the automated features had practically no impact on their activities, the exception being the Students.

Indifference (Birders). The Birders' view on Jaiku's location automation is best described as indifference or neglect. They did not bring up any particular privacy concerns, but found little use for the automatic disclosure either. This was probably because they did not have close ties and there was little risk in accidentally disclosing personal information. Simply put, the Birders were so distant that it did not matter if the rest of the user group knew their location and could follow them.

Initial Conflict and Withdrawal (Hipsters). The majority of Hipsters had a negative first impression about the concept of Jaiku, and one reaction was: "I can't lie anymore." The automatic location disclosure conflicted with the group's structure of privacy. They were not used to knowing about each other in real-time on a daily basis. One of the few active Hipster users occasionally checked if his girlfriend was at home, and if she was, he might call her because he knew it meant she was available. This user said: "With Jaiku you can get a light touch with your friends." However, because many of the users were not logged in all the time, the automatic location disclosure had little value and could not be used reliably. Thus, the negative impacts that were predicted did not realize among Hipsters because they simply neglected the system or switched it off.

Part of the Communication Toolbox (Students). The Students were the only one exhibiting a clear benefit from Jaiku's automation. When using Jaiku, they used the automatic features for coordinating shared activities and to get information about each other's whereabouts. They used automatic location disclosure, for example, when participant A noticed that participant B was nearby and A sent a Jaiku message to he suggest a joint lunch. They also learned to use location disclosure for checking the other's availability to receive calls:

"For many it says like " [preparation course]". That tells they are there, you don't want to call them then. You can call them later" $-\mathrm{M} 18$

Jaiku became a part of their communication toolbox, using it to draw conclusions about which communication channel to use in different situations and coordinate mobility. For example, they looked at the other's automatic information to decide whether a SMS or a call would be appropriate. Despite being the most involved group, the Students showed no strong privacy concerns. However, they did speculate that if their parents or their boy/girlfriends belonged to the group, they would use Jaiku differently.

\section{FINDINGS PART 3: UNDERSTANDING THE LOGIC OF AUTOMATION}

We interviewed the users about their understanding of the concept of automation in Jaiku. The question of users' understanding is a classic human factors topic. As previous sections have explained, the workings of location automation 
in Jaiku are not obvious (even to researchers who must deduce the logic from observable behavior of the application), simply because of the multiple hidden layers of processing that affect a perceivable outcome. In comparison to human factors studies, the incidents we report convey the idea that the logic of automation mainly became problematized by users when the automation prevented them from achieving their communicative and social ends. In other words, they did not spontaneously explore the logic, out of pure curiosity or as part of familiarizing with the system.

A methodological note before reporting the results. Our participants were initially introduced to how locations are diffused in Jaiku, and this must of course have influenced their capacity for understanding. Despite this introduction, which is more than average users will go through, several problems emerged. With less instruction, we surmise that the problem of understanding would be more pronounced.

\section{A. "You are still home?": Problems with diffusion}

Despite this introduction, some users forgot the basics and did not know if location names are diffused for all Jaiku users or only among the contacts. Jaiku does not make transparent its logic of diffusion and, unless told by a third party, it is very hard for a user to figure out the diffusion logic herself. Some also confused the difference between presence line and location information in Jaiku. Two participants mentioned how they first wrote location names to Jaiku's presence line because they did not understand that writing in the presence line is not similarly automated as the location line.

The problem with diffusion was worst in the Hipsters group. A couple living about one mile from each other had both named their home location in Jaiku as "home," and when the woman visited the man's home, they noticed that her Jaiku also showed "home." They thought that the accuracy of location technology was so poor that it did not change the woman's location. However, this was an artifact of the way Jaiku propagates location labels between users. As was discussed earlier, the diffusion of Jaiku's location information sparked strong opinions and privacy concerns.

\section{B. "Still in a meeting?": Misinterpreting timeliness}

There were also problems with inferring from Jaiku's UI if information is up to date.

For every contact Jaiku's main menu shows when the user last used Jaiku (e.g. looked at contacts' information) and the current presence line (status message), [[last time of activity]:[presence line]], e.g. "11 hours ago: going home." This does not mean the presence line is updated but only that the user has done something with Jaiku.

One Hipster told looking at a friend's presence line," 1 hour ago: in a $2 \mathrm{~h}$ meeting,". However, it later turned out the status was already several hours old but the time " 1 hour ago" was updated the previous time the user was online in the system. Jaiku has information on how old a status update is, but that has to be viewed from a different menu. Unsurprisingly, this caused problems in knowing how old or new the information was. A simple remedy is to indicate already in the user interface if location data are obsolete.

\section{C. "It was really S.F.": Accuracy and reliability}

In Jaiku the location information is acquired using the GSM network cell tower identification code (cellID). Jaiku has three location categories: Country, City, Neighborhood. Country is automatically fetched from the phones country code and City and Neighborhood are user created names for specific cellIDs. However, the system gives the user no information about the real accuracy of the location. Users have no knowledge of the fact that GSM cellIDs determine the accuracy, neither can they observe the boundaries of GSM cells in their environments.

Not surprisingly, the users were often confused with the accuracy of automatically disclosed information. The majority of Students mentioned that it was unclear how large the geographic span of a named area is:

"I don't know how large the geographic span of a named location is. There is this city/region, but how large is it in reality? "-M18

This user-initiated "repair" of accuracy, in addition to function of enhancing the status messages' communicativeness, were main reasons for the result (reported above) that $40 \%$ of location labels referred to finer geographical areas than what the cellID-based system allows.

\section{D. "In Having Coffee": Making Sense of Intended uses}

The users also had problems in understanding Jaiku's main menu that shows the contacts' location and presence line (status message) in format: in [location]: [presence line]. This is intended to show the users what their contacts are doing and where they are doing it. Also both location and presence information have different input menus where the user the user can write and submit the information.

Having problems understanding the logic, timeliness, and accuracy of automatic location data, some of the participants were struggling to see its purpose in Jaiku. As said above, they sometimes wrote their locations in the presence line, and also, wrote non-location information in the location line (e.g., "having coffee", which shows in Jaiku as "in having coffee"), mixing the intended purposes of the two. Although the latter was possibly done intentionally it most likely obscured the norms of use within a group.

\section{VIII.DISCUSSION}

This study is the first reported empirical study focusing on automation in location-based services. It showed how the automation related questions are essential factors affecting user experience of location based systems, and should be taken seriously when designing mobile social applications.

The results reveal both "classic" human factors problems with the automation's logic and novel issues related to the fact that location automation at times compromised their control of social situations. Relating to the first class of problems, the users did not always understand the geographic area covered by GSM cell IDs, the timeliness of the data when other users were off-line, or the purpose of real-time location disclosure. 
Relating to the second class of problems, we see many effects from privacy to abandonment of the application. Only one group found real benefit from Jaiku - the Students who used it for coordinating shared activities and ad hoc encounters. In these pursuits, the automated location information was useful but often insufficient. In $55 \%$ of time the members of this group labeled locations more accurately than the GSM cellbased technology really supported. The two other groups exhibited indifference and withdrawal toward Jaiku, but their reasons were different. The Hipsters were initially worried about privacy problems and generally felt that they do not need such a channel as they already interact with each other often enough. They rarely logged in and used it mainly to check others' availability. Although the common activity of Birders was spatial by nature, they did not have simultaneous collective efforts, and they did not know each other outside the interest groups, with the result that the automatic information was simply uninteresting and need not to be controlled. Automation was a non-issue to them. These differences highlight the importance of needs, activities, and structures of the intended user groups as factors for acceptance of automation.

In reference to the three questions we set in the beginning, our observations can be summarized as the following set of claims about automation in mobile social applications.

Use of automated features in mobile social applications:

1. Automation can threaten accountability of actions. When automated data are mixed with manual data in the UI, the danger is that the communicative functions of tags and manual location labels are lost.

User response to automation:

2. The negative effects of automation at the level of the group include neglect in the face of useless automation, and withdrawal in the face of a too threateningly strong model of automation.

3. Privacy concerns are felt but do not necessary actualize due to the measures the users can take both inside and outside the system.

4. The nature of shared activities, and the usefulness of the automated information therein, shapes whether automation will be useful, useless, or annoying. The level of automation, and its content, should be chosen according to what is known about these activities.

Users' understanding of the logic of automation:

5. The negative effects of automation on the individual reflect the classic findings of human factors. Particularly, ignorance of automation, misunderstanding of its operating logic, post hoc "repairs," repetitive behavior (re-sending location labels to ensure they appear), and eventually shutting down of the system can emerge.

\section{A. A thought experiment}

A broader point we have made in the paper is that Sheridan's levels of automation framework is useful for thinking about location automation. The framework shows that for any mobile social application there are numerous options on how to implement different features. Importantly, as a consequence of changing an automated solution from one level to another, interaction and the whole concept can change. If we make a thought experiment and take Sheridan's all seven levels for each of the four processes in Jaiku, we get $7 \times 4$ matrix. For each of the four stages we have seven different ways of implementing the automation, and hence, get 2,401 different ways of implementing location automation. In our case, the production of location information was divided into four processes. Other systems may have simpler or more complex constituent structures. The breakdown of different automation design choices shows that the "design space" is anything but simple and trivial

The results of the study and the previous thought experiment about the complex design space of automation in social mobile application also makes visible two assumptions about automation that may be problematic. First, productivity should not be seen as the dominant goal when designing automation. Indeed, Jaiku's automation may have increased "productivity" of Students by creating location information without burdening the user, but as the data shows, this may give raise to a myriad of other (deeper) problems. Automated processes are intertwined with the social practices, attitudes and prejudices of the users. However, if the system was more flexible, and users could change the levels of automation themselves, it might make the system more suitable to various types of users and groups. For, example, people inside a user group might have different norms related to specificity of disclosed location. The user should be allowed to set the level of how specifically her location is disclosed. This should also be independent on how specifically the user's contacts show their locations or how specific the location names named and diffused by them are.

Second, the automation's "locus" in this context is not between the human operator and the operated machine as is common in human factors. In mobile social applications the key processes occur between humans. In effect, these processes are constituted by elements outside the system's influence. The paradox of automation in this context is that it should automate a process, which by nature cannot be fully automated. Importantly, changing the model of automation will change how well the system integrates into to the social practices of the user group. Especially non workplace related social environments, such as group of friends, might vary in complexity and formalization. As this study showed, when designing a group communication system like Jaiku, the target audience should not be categorized as vaguely as a group of friends. Both the Students and the Hipsters were a group of friends but appropriated to the systems very differently. A major reason for that was the fact that the automated features of Jaiku suited the Students but were unsuitable for the Hipsters. This calls for including proper user trials as integral part of system evaluation.

We hope that our study has opened a door in designing better applications and middleware for location-based 
services. We hope it showed how important it is to systematically take into account the various levels of automation when designing the processes of location-based applications. In a social context of use, the automation of location information should facilitate the user to easily communicate with other people. The goal of automation is to make in the use of location information in everyday social contexts flexible, transparent, and understandable. The goal of automation is not primarily to increase effectiveness or accuracy.

Our study also points out that the design of automation in location-based services is not a sole matter of user interface design. The design decisions made in the middleware or backend of the system can be critical for what the user interface is able to display and the users fathom.

\section{ACKNOWLEDGMENT}

We thank Sauli Tiitta and Mika Raento for support in organizing the study, and Jamie Munger and anonymous reviewers for comments on manuscript.

\section{X.REFERENCES}

[1] Ahern, S., King, S., Naaman, M., Nair, R. and Yang, J.H.I. ZoneTag: Rich, Community-Supported Context-Aware Media Capture and Annotation. In Proc. CHI 2007, ACM Press (2007).

[2] Altman, I. The Environment and Social Behavior: Privacy, Personal Space, Territory, and Crowding. Brooks/Cole Publishing Company, Monterey, CA, USA, 1975.

[3] Ames M., Naaman M. Why we tag: motivations for annotation in mobile and online media. In Proc CHI 2007, ACM Press (2007), 971-980.

[4] Arminen I., Social functions of location in mobile telephony, Personal and Ubiquitous Computing 10, 5 (2006), 319-323.

[5] Barkhuus L., Brown B., Bell B., Sherwood S., Hall M. and Chalmers M. From awareness to repartee: sharing location within social groups. In Proc CHI 2008, ACM Press (2008), 497-506.

[6] Barkhuus, L., and Dey, A. Location-based services for mobile telephony: A study of user's privacy concerns. In Proceedings of the INTERACT, $9^{\text {th }}$ IFIP TC13 International Conference in HumanComputer Interaction. (Zürich, Switzerland, September 2003).

[7] Brown, B., Taylor, A., Izadi, S., Sellen, A., Kaye, J. and Eardley, R. Locating Family Values: A Field trial if the Whereabouts Clock. Ubicomp 2007, 354-371.

[8] Consolvo, S., Smith, I., Matthews, T., LaMarca, A., Tabert J., Powledge, P., Location disclosure to social relations: why, when, \& what people want to share, In Proc CHI 2005, ACM Press (2005), 81-90.

[9] Harper, R., Looking at ourselves: an examination of the social organisation of two research laboratories. In Proc CSCW 1992, ACM Press (1992), 330-337.

[10] Iachello, G., Smith, I., Consolvo, S., Abowd, G. D., Hughes, J., Howard, J., Potter, F., Scott, J., Sohn, T., Hightower, J. and LaMarca, A. Control, Deception, and Communication: Evaluating the Deployment of a Location-Enhanced Messaging Service. In Proc UbiComp 2005, ACM Press (2004), 213-231.

[11] Jiang, T., Wang, H. J., and Hu, Y. 2007. Preserving location privacy in wireless lans. In Proceedings of the 5th international Conference on Mobile Systems, Applications and Services (San Juan, Puerto Rico, June 11 - 13, 2007). MobiSys '07. ACM, New York, NY, 246-257.

[12] Junglas, I. A. and Watson, R. T. 2008. Location-based services. Commun. ACM 51, 3 (Mar. 2008), 65-69.

[13] Laurier, R. Why people say where they are during mobile phone calls. Environment and Planning D: Society \& Space (2001), 485-504.

[14] Ling, R., Yttri, B. Hyper-coordination via mobile phones in Norway, Perpetual contact: mobile communication, private talk, public performance. Cambridge University Press, New York, NY, USA 2002, 139-169.
[15] Oulasvirta, A., Petit, R., Raento, M., and Tiitta, S. Interpreting and acting on mobile awareness cues. Human-Computer Interaction 22 (2007), 97-135.

[16] Palen, L., Dourish, P. Unpacking "privacy" for a networked world, In Proc CHI 2003, ACM Press (2003), 129-136.

[17] Raento, M., Oulasvirta., A., Petit, R. and Toivonen, H. ContextPhone: A prototyping platform for contextaware mobile applications. IEEE Pervasive Computing 4, 2 (2005), 51-59.

[18] Sarvas, R., Herrarte, E., Wilhelm, A. and Davis, M. Metadata creation system for mobile images. In Proc MobiSys 2004, ACM Press (2004), 36-48.

[19] Sellen, A., Rogers, Y., Harper, R., Rodden, T. Reflecting human values in the digital age. Communications of ACM 52, 3 (2009), 58-66.

[20] Sheridan, T. Telerobotics, automation, and human supervisory control, MIT Press, Cambridge, MA, USA 1992.

[21] Sheridan, T., Parasuraman, R. Human-Automation Interaction, Review of Human Factors and Ergonomics, 1 (2006), 89-129.

[22] Want, R., Hopper, A., Falcao, V., and Gibbons, J. The Active Badge Location System. In ACM Transactions on Information Systems (TOIS) 10, 1, ACM Press (1992), 91-102

[23] Youssef, M., Youssef, A., Rieger, C., Shankar, U., and Agrawala, A. 2006. PinPoint: An Asynchronous Time-Based Location Determination System. In Proc MobiSys 2006. ACM, New York, NY, 165-176. 